\title{
Evolution of the modern chemotherapy of leprosy
}

\author{
L LEVY \\ Visiting Professor, Department of Comparative Medicine, Hebrew \\ University-Hadassah Medical School, Jerusalem, Israel \\ Chairman, Steering Committee, Scientific Working Group on the \\ Chemotherapy of Leprosy (THELEP), UNDP/World Bank/WHO \\ Special Programme for Research and Training in Tropical Diseases, \\ Geneva, Switzerland
}

Certainly, the modern chemotherapy of leprosy has depended upon the availability of potent drugs. It could be argued that new, more potent, drugs would have been introduced into the chemotherapy of leprosy without the prior development of laboratory means by which to assess their antimicrobial potency and their efficacy in the chemotherapy of leprosy. However, the history of the evolution of modern chemotherapy is, in large part, the history of the development of more sensitive and more precise means of measuring the potency of drugs against Mycobacterium leprae, and of measuring the response of patients with multibacillary (i.e. lepromatous and borderline-lepromatous) leprosy to chemotherapy. In other words, the availability of potent drugs was a necessary but not a sufficient condition.

Because assessment of the efficacy of antimicrobial chemotherapy requires measurements made on the microorganisms themselves, the chemotherapy of paucibacillary (i.e. non-lepromatous) leprosy will not be considered. As the term implies, patients with paucibacillary leprosy ordinarily have such small populations of $M$. leprae that the effects of antimicrobial drugs on the organisms cannot be studied directly.

Three phases of the evolution of the modern chemotherapy of leprosy may be identified. The first phase, comprising the 20-year period 1940-60, was one of attempting to screen drugs for antimicrobial potency and to assess the efficacy of drugs in therapy, with the aid of imprecise techniques of measurement. The second phase was ushered in by Shepard's descriptions of his technique for cultivating $M$. leprae in the mouse foot pad ${ }^{46,47}$ and was concluded in 1974 by the demonstration of Rees and his colleagues ${ }^{16,41,64}$ that $M$. leprae possess the 
capability of persisting. This 15 -year period also saw the detection of drug-resistant $M$. leprae. Although at this moment the third phase of the evolution of modern chemotherapy has endured for only 9 years, it is already possible to characterize it as a period of increasing concern over the apparently increasing incidence of relapse caused by the emergence of $M$. leprae resistant to dapsone, the recognition that persisting $M$. leprae do not pose the great danger to multibacillary patients that had been anticipated, and a change in emphasis from determination of the antimicrobial potency of individual drugs to investigation of the chemotherapeutic efficacy of combined drug regimens.

It appears useful to consider the evolution of modern antileprosy chemotherapy under the two main headings of 'drug-screening' and 'evaluation of chemotherapeutic efficacy'.

\section{Drug-screening}

Usually, drugs are screened for antimicrobial potency by exposing the target organisms to the drugs in cell-free culture. It has been necessary to screen drugs for activity against Mycobacterium leprae by other means. This was first accomplished in a necessarily imprecise and sometimes hazardous way, by employing the drugs in the treatment of leprosy patients. Because there existed no more sensitive means of assessing the eff ects of treatment on the patients' disease, potency of drugs was measured in terms of clinical response and the decrease of the bacterial index (BI). The series of multicentre trials conducted by the Leonard Wood Memorial during the 1950 ' $\mathrm{s}^{7-13,59}$ exemplifies this method of testing the potency of drugs. These trials necessitated the exposure of large numbers of patients to the hazards of treatment by untried drugs, or, because it was at first necessary to employ placebo-treated controls, to the hazards of remaining untreated. In addition, these methods were insensitive; when a valid measurement was obtained, it was all-or-none in character.

Following Shepard's reports ${ }^{46,47}$ of the multiplication of $M$. leprae in the foot pad of the immunologically normal mouse, and his first demonstrations in this system of the potency or lack of potency against $M$. leprae of the drugs then commonly used in the treatment of tuberculosis and leprosy, ${ }^{50,51}$ this has become the accepted means of measuring the potency of antileprosy drugs. In this system and its variants, ${ }^{4,48,49}$ the results of testing drugs of established potency are sufficiently reproducible, that it is no longer necessary to screen drugs in patients. Moreover, it has been possible to measure the minimal inhibitory concentrations of many drugs, and to distinguish between primarily bactericidal and primarily bacteriostatic effects.

Although application of $M$. leprae infection of the foot pad of the normal mouse has represented a tremendous advance in screening drugs, the technique is not without its limitations. Large quantities of drug and many mice are required. 
Moreover, because the mouse is interposed between the drug and the organism, there is always the risk that active compounds will be found inactive and inactive compounds active, because of metabolic alterations of the compounds by the mouse.

In the absence of techniques for cultivating $M$. leprae in vitro, workers have been forced to consider the use of cultivable mycobacterial species as surrogates of $M$. leprae. At first, this was done uncritically, and potency of a drug against, for example, M. tuberculosis or M. marinum was accepted as presumptive evidence of potency against $M$. leprae. Modern workers have proceeded far more thoughtfully. Arguing that, among the numerous cultivable mycobacterial species, some must resemble $M$. leprae more closely than do others in terms of a specific target enzyme or enzyme system, they have begun by screening species for sensitivity to a drug equivalent to that of $M$. leprae, or for a pattern of sensitivity to drugs of a specific class like that of $M$. leprae.

Thus, Seydel has employed as a surrogate of $M$. leprae the cultivable ' $M$. $l u f u$ ' ${ }^{36}$ a species that is highly susceptible to the antimicrobial action of dapsone. ${ }^{45}$ And Pattyn has employed a battery of strains of rapidly growing mycobacterial species, principally strains of $M$. phlei, that appeared to exhibit the same pattern of sensitivity to rifampicin and several of its analogues as did M. leprae. ${ }^{27,28}$

Another approach to drug-screening without interposing a mammalian host between drug and $M$. leprae has involved inhibition of the uptake of radioactively labelled substrates by $M$. leprae in short-term culture. Khanolkar ${ }^{1,18,19}$ has worked with $M$. leprae incubated in a modification of Murohashi's medium, whereas Nath and her coworkers ${ }^{26,37,44}$ have employed $M$. leprae-infected macrophage monolayers.

\section{Chemotherapeutic efficacy}

Having available a number of potent drugs, it is necessary to assess their efficacy in the chemotherapy of leprosy, in order to learn best how to employ them. These two measurements - of potency and of efficacy - are quite different. Typically, potency is measured against a small number of Mycobacterium leprae, in terms of killing or of preventing the multiplication of a small number of organisms, all of which are genotypically and phenotypically identical. On the other hand, chemotherapeutic efficacy, at least in the treatment of multibacillary leprosy, is measured against much larger numbers of organisms, larger by 6-8 orders of magnitude, when disease has been established, and there has developed genetic and physiologic diversity among the members of the bacterial population.

In parallel with the developments in drug-screening, our ability to measure chemotherapeutic efficacy has developed greatly in the 22 years since the introduction of the mouse foot-pad technique. In the absence of an experimental model of the lepromatous patient, however, chemotherapeutic efficacy must be measured in patients. 
PHASE ONE

Thus, Faget and his coworkers at the National Hansen's Disease Center in Carville, studied the efficacy of a series of sulphones, beginning with glucosulphone $\left(\right.$ Promin $\left.^{\circledR}\right) .{ }^{15}$ These investigators and others working at that time had available to them three imprecise means by which to measure the efficacy of leprosy chemotherapy: 1, the response of the patients as assessed by repeated clinical observation; 2 , the decrease of the $\mathrm{BI}$; and 3 , the rate of relapse after withdrawal of chemotherapy.

As was the case when antimicrobial potency was measured by administering drugs to patients (in fact, no distinction was made at that time between measurements of potency and those of efficacy), the measurements required exposure of large numbers of patients to unknown risks for long periods of time. In addition, the measurements were insensitive. They were applied to events far removed from the critical event-the encounter between organism and drug. Measurement of the $\mathrm{BI}$ is the only method of the three that directly involves $M$. leprae. However, the decrease of the BI depends only secondarily on the rate at which $M$. leprae are killed during effective chemotherapy, and reflects primarily the degradation of already killed $M$. leprae, a phenomenon which is not an immediate consequence of bacterial killing. ${ }^{63}$ Moreover, so long as $M$. leprae are being killed in the course of effective chemotherapy, the rate of decrease of the BI bears no relationship to the rate of killing. On the contrary, the rate of decrease of the BI appears to be constant, approximating $1 \log _{10}$ unit $(90 \%)$ per year, at least during the first years of therapy of LL leprosy. ${ }^{42,52}$ Not only is the measurement of the BI an insensitive means of assessing chemotherapeutic efficacy. It is also imprecise, because the error of the measurement in the individual patient may be as large as the change expected during effective treatment.

Although the yield of the first 20 years of studies of leprosy chemotherapy was disappointingly small, this first phase saw the establishment of the efficacy of dapsone as monotherapy.

\section{PHASE TWO}

\section{The morphological index}

The experimental basis for modern leprosy chemotherapy depended upon the development of two laboratory techniques that rendered possible the direct study of the effects of antimicrobial drugs on M. leprae. The first of these techniques, measurement of the morphological index (MI), was based on the morphological changes of the organism that accompany its death. After earlier workers had called attention to the morphological changes undergone by $M$. leprae during treatment of leprosy patients, ${ }^{6,22,25}$ Rees and his coworkers ${ }^{39,40}$ found evidence that morphologically imperfect $M$. leprae were, in fact, dead, and employed the 
decrease of the MI during treatment as a means of measuring therapeutic efficacy in a series of trials of chemotherapy of multibacillary leprosy carried out in the Sungei Buloh Leprosarium in Malaysia.

The trials at Sungei Buloh established that, during treatment of previously untreated patients with lepromatous leprosy with dapsone alone in dosages totalling from 300 to $600 \mathrm{mg}$ weekly, the MI decreased to baseline values within 3-6 months. ${ }^{62,63}$ The addition of macrocylon ${ }^{60,63}$ or ditophal ${ }^{62}$ to the dapsone did not result in a more rapid decrease of the MI. Clofazimine, $300 \mathrm{mg}$ daily, 6 days per week, ${ }^{35}$ and $100 \mathrm{mg}$ twice weekly, ${ }^{61}$ and dapsone, $50 \mathrm{mg}$ twice weekly, ${ }^{31,34}$ appeared as effective by this criterion as the larger, conventional dose of dapsone. This change of the MI in the course of effective chemotherapy was consistent with death of $M$. leprae, and thus provided the first more-or-less direct measure of the effect of antimicrobial drug on the organism. Thus, the MI permitted one to recognize effective antileprosy drugs during clinical trials of only a few months' duration involving only small numbers of patients, a goal previously attainable only by clinical trials enduring at least 1 year and involving several tens of patients as a minimum.

However, the change of the MI appeared rather insensitive as a measure of response to antimicrobial therapy. The rate of decrease of the MI appeared no less rapid during treatment with much smaller dosages of dapsone and clofazimine as during treatment with these drugs administered in full dosage. One explanation for this insensitivity is suggested in the report of the Committee on Experimental Chemotherapy. ${ }^{5}$ Using, instead of the MI, the more rigorously defined 'solid ratio' of Shepard, ${ }^{23}$ it is apparent that the average patient with lepromatous leprosy presents before any treatment with a solid ratio no greater than 10 per 100 , i.e. no more than $10 \%$ of his organisms are solidly staining and, therefore, presumed infective for mice (viable). The patient may harbour as many as $10^{11}(100,000,000,000) M$. leprae. Because it is not feasible to examine many more than 100 individual organisms in the course of the measurement, the solid ratio will have fallen to $<1$ per 100 organisms after only $90 \%$ of the viable $M$. leprae have been killed. At this time the patient still harbours as many as $10^{9}$ viable organisms. Thus, the rate of loss of viable $M$. leprae, in terms of the decrease of the solid ratio, is measured only during a very small segment of the patient's response to chemotherapy. A second explanation of the insensitivity of the solid ratio is that the death of $M$. leprae is probably not rate-limiting; rather, the rate-limiting step appears to be a secondary change of morphology of dead bacilli that occurs at a more-or-less constant rate, which may be slower than the rate at which the bacilli are killed. Thus, although $M$. leprae were killed much more rapidly during therapy with rifampicin than during dapsone monotherapy, as measured by mouse inoculation, the solid ratio decreased at the same rate in both groups of patients. ${ }^{54}$ 


\section{The mouse foot-pad technique}

The application of Shepard's mouse foot-pad technique, the second of the two new laboratory techniques, to the measurements of the rate at which $M$. leprae are killed during effective antimicrobial treatment of the patient with lepromatous leprosy, first reported in $1968,{ }^{52}$ provided a more quantitative and sensitive means of evaluating the efficacy of chemotherapy by individual drugs. In this application, mouse inoculation is employed in much the same way that sputum culture is employed in assessing the efficacy of an antituberculosis drug. A biopsy is performed before the start of treatment, a $50-100 \mathrm{mg}$ specimen providing $10^{6}-10^{8}$ organisms if the patient is lepromatous and previously untreated. The specimen is minced and homogenized, and an aliquot is spread over the measured area of a microscope slide for acid-fast staining and direct enumeration of the $M$. leprae. The bacterial suspension is diluted to provide an inoculum of from 5,000 to $10,000 \mathrm{M}$. leprae per foot pad, and the hind foot pads of from 10 to 20 normal mice are inoculated. According to Shepard's technique, one mouse is sacrificed each month, beginning about 3 months after inoculation. The inoculated hind foot pad is processed for histopathological examination, and paraffin sections are stained by an acid-fast stain. The presence in a section of a lesion filling at least one-fourth of a $\times 540$ microscope field with brightly staining organisms indicates the end of the 'incubation period', and is the signal for performing a harvest. The tissues, usually of four inoculated foot pads, are pooled, minced, and homogenized, and aliquots of the resulting bacterial suspension are spread uniformly over measured areas on slides, fixed and stained, and the $M$. leprae are enumerated. The generation time is then calculated as if multiplication of $M$. leprae had occurred at a constant rate from the day of inoculation until the day of harvest. The killing of $M$. leprae during therapy is indicated by progressive increases of the values for the incubation period and the generation time.

Employing this technique, a series of clinical trials was carried out in San Francisco and in Cebu, the Philippines. These trials established that, on the average, $M$. leprae recovered from patients lost their infectivity for mice after: 100 days of treatment with dapsone, 50-100 mg daily;,2, 20,52-4 150 days of treatment with clofazimine, 100-200 mg daily or $100 \mathrm{mg}$ three times weekly; ${ }^{3,20}$ longer than 150 days with acedapsone (4,4'-diacetamidodiphenylsulphone, DADDS), 225 mg intramuscularly every 77 days, ${ }^{53}$ or clofazimine, $600 \mathrm{mg}$ every 2 weeks or 1,200 mg every 4 weeks; ${ }^{3}$ and within a few days of single 1,200 or $1,500 \mathrm{mg}$ doses or daily $600 \mathrm{mg}$ doses of rifampicin..$^{2,21,54,55}$ In a much less extensive study, the anti- $M$. leprae activity of ethionamide was established (Levy L, Shepard CC, Fasal P, unpublished results).

Thus, the second phase of the evolution of leprosy chemotherapy was marked by establishment of the efficacy in both absolute and relative terms of chemotherapy of multibacillary leprosy by dapsone, acedapsone, clofazimine, rifampicin and ethionamide, administered as monotherapy. These results also 
pointed up the limitations of the mouse foot-pad technique as a measure of response to antimicrobial therapy. One limitation of the sensitivity of the technique is imposed by the maximal number of $M$. leprae that may be inoculated and be seen to have multiplied, if multiplication has indeed occurred. In immunologically normal mice, multiplication ceases when the number of $M$. leprae approaches 1-2 million per foot pad; the mice then appear to have mounted an effective immune response. Inocula larger than 100,000 per foot pad fail to give rise to multiplication, and inocula must be no larger than 10,000 per foot pad, if multiplication is to be recognized reliably. The failure of multiplication in mice, each inoculated with 10,000 M. leprae, implies only that the inoculum included fewer than 1:10,000 viable organisms, and tells us nothing about the remaining $M$. leprae, as many as $10^{7}$ of which may be viable. A second limitation is imposed by the great rapidity of the bactericidal action of rif ampicin. By inoculating mice, one cannot measure a rate of killing of $M$. leprae faster than that produced by rifampicin alone, as might be the case if a second active drug were used in combination with rifampicin.

\section{PHASE THREE}

\section{Dapsone resistance}

The third and current phase of the evolution of the modern chemotherapy of leprosy had its beginnings in the first reports by Pettit and Rees ${ }^{32,33}$ of relapse of lepromatous leprosy associated with the emergence of dapsone-resistant $M$. leprae, and in the first reported demonstration, also by Waters, Rees and their colleagues ${ }^{64}$ of persisting (surviving, fully drug-susceptible despite apparently adequate chemotherapy) $M$. leprae. The recognition of these two phenomenadrug resistance and microbial persistence - emphasized the shortcomings of the chemotherapy (almost always monotherapy) in use at the time.

Pettit \& Rees ${ }^{32}$ demonstrated at Sungei Buloh 3 patients with lepromatous leprosy whose disease had relapsed after 13-15 years of apparently adequate dapsone therapy; as measured by clinical observation and determination of the MI, these patients failed to respond to dapsone administered by injection in a dosage of $300 \mathrm{mg}$ twice weekly, despite the achievement of adequate blood dapsone concentrations. The $M$. leprae isolated from these 3 patients by mouse inoculation were not inhibited from multiplying by administration to the mice of diet containing dapsone in a concentration of $0.1 \mathrm{~g}$ per $100 \mathrm{~g}, 1000$-fold the minimal effective dosage of dapsone for $M$. leprae in the mouse.

Although dapsone resistance had occasionally been suspected before this study was carried out, these were the first proven cases. In their discussion, the authors stated that 'there are ... very few cases which relapse under treatment'. The current situation, which represents a dramatic change during the 18 years since this report appeared, has recently been exhaustively reviewed. ${ }^{24,29}$ Evidence 
of the magnitude of the threat to leprosy control efforts posed by the apparently increasing prevalence of secondary resistance to dapsone was furnished by reports of high prevalence of primary resistance to dapsone. Estimates of the prevalence of primary dapsone resistance have varied from 3.3 per 100 in Cebu, Philippines, ${ }^{17}$ and 35 per 100 in Chingleput, South India and Bamako, Mali, ${ }^{58}$ to 55 per 100 in Addis Abada. ${ }^{30}$

\section{Microbial persistence}

The demonstration in 1966 by Rees ${ }^{38}$ that T-cell depleted (adult-thymectomized, whole-body-irradiated, and bone-marrow-reconstituted; T900R) mice permitted multiplication of $M$. leprae to a higher limit than did normal mice provided the basis for an important advance in the assessment of chemotherapeutic efficacy. T900R mice regularly permit multiplication after inoculation of as many as $100,000 \mathrm{M}$. leprae per foot pad. Inoculation of these immune-deficient rodents with 100,000 $M$. leprae recovered from skin biopsy specimens obtained at intervals from multibacillary patients during treatment, although theoretically capable of greater sensitivity than the technique employing smaller inocula in normal mice, has not permitted us to measure chemotherapeutic efficacy with greater sensitivity. But the problem is no longer a limitation of the technique, but rather an important feature of multibacillary leprosy - the ubiquity of persisting M. leprae.

The first evidence that $M$. leprae are capable of persisting was reported by Waters, Rees and their colleagues. ${ }^{64}$ Seven of 12 lepromatous patients who had completed at least 10 years of continuous dapsone therapy were found to harbour small numbers of $M$. leprae capable of infecting mice; three strains were passaged and found to be fully susceptible to dapsone. The detection of persisting M. leprae was reported subsequently in patients with lepromatous leprosy af ter treatment with acedapsone for 3 or 4 years ${ }^{43}$ after treatment with rifampicin as monotherapy in a daily dose of $600 \mathrm{mg}$ for from 2 to 5 years, ${ }^{41}$ and after treatment with the combination rifampicin plus dapsone, each drug administered in a full daily dose, for 6 months. ${ }^{16}$

The identification of surviving M. leprae as persisters appears best established by the results of acedapsone therapy reported by Russell and his coworkers. ${ }^{43}$ In this study, the clue to the continued presence of significant proportions of viable organisms was the finding of solidly-staining organisms in the smears of one patient after 3 years of treatment, and in the smears of additional patients after treatment for 4 years. After the solid organisms had been seen, skin lesions were biopsied and mice were inoculated, with the result that normal mice were infected with $M$. leprae that proved to be susceptible to dapsone. Although mice had not been inoculated earlier in this study, it had been reported ${ }^{53}$ that, as the result of another trial of acedapsone, $M$. leprae became non-infective for mice after about 6 months of acedapsone treatment. Moreover, solidly staining $M$. leprae had 
disappeared by the end of the first year of treatment. The absolute number of viable organisms appears to have reached a minimum after 2 years of treatment; thereafter, the total number of $M$. leprae decreased still further, unmasking the persisting organisms, the proportion of which actually increased.

The identification of drug-susceptible $M$. leprae that survive some period of treatment as persisters may appear somewhat arbitrary. However, there arose the need to explain the apparent paradox that, for example, $M$. leprae are rendered non-infective for normal mice after treatment for only a few days with daiiy rifampicin, ${ }^{21,54,55}$ whereas viable (i.e. mouse-infective) organisms may again be detected after 2 years of this same therapy. ${ }^{41}$ The explanation lies partially in the insensitivity of the normal mouse; T900R mice are capable of detecting smaller proportions of viable $M$. leprae than may be detected in normal mice. These data suggest that, despite continued chemotherapy, $M$. leprae are not killed at the same rate as that measured initially. It is the remaining viable organisms that are identified as persisters.

The demonstrations that current leprosy chemotherapy was incapable of eradicating the $M$. leprae infection in patients with lepromatous leprosy, who are known to be immunologically deficient, appeared to confirm the widely-held belief, largely based on anecdotal evidence, that patients with lepromatous leprosy whose chemotherapy was stopped were very likely to suffer relapse, in the course of which they would become infectious to their contacts. Based on this belief, very long-term and even life-time chemotherapy of lepromatous leprosy was usually recommended (see, for example, ${ }^{65}$ ). As a result, leprosy control programmes have become burdened with the supervision of patients for periods of many years' duration, an impossible burden for all but the best-funded of programmes. The addition to such a programme of a second drug, almost by definition more expensive and more toxic than dapsone, has generally been impossible.

The current phase of the development of an effective chemotherapy of leprosy may be considered to have begun, therefore, from the recognition of the need to prevent the emergence of drug-resistant $M$. leprae, and to minimize persisting populations of $M$. leprae. More rapidly effective combined drug regimens are under study among patients with previously untreated lepromatous leprosy in formal clinical trials, in which persisting $M$. leprae are systematically sought. Sponsored by the Chemotherapy of Leprosy (THELEP) Scientific Working Group of the UNDP/World Bank/WHO Special Program for Research and Training in Tropical Diseases, ${ }^{57}$ controlled clinical trials of combined drug regimens have been undertaken in Bamako, Mali, and in Chingleput, South India. In each centre, a 'maximal' regimen (in Bamako, daily rifampicin, prothionamide and dapsone, each in full dosage, for 2 years; in Chingleput, daily rifampicin, clofazimine and dapsone for 2 years) is compared with a 'minimal' regimen (daily dapsone for 2 years, together with a single, initial $1500 \mathrm{mg}$ dose of rifampicin in both centres) and a regimen intermediate in terms of the duration of 
combined chemotherapy (in Bamako, a 3-month course of prothionamide and dapsone administered daily and rifampicin weekly in a $900 \mathrm{mg}$ dose, followed by daily dapsone alone for 21 months; in Chingleput, a single initial dose of rifampicin daily clofazimine and dapsone for 3 months, followed by dapsone alone for 21 months). These regimens were selected, not because they were considered optimal, but rather to learn if the 'stronger' regimens would effect a greater reduction of the population of persisting $M$. leprae. In fact, this does not appear to have occurred. Persisting $M$. leprae have thus far been detected in very few of the patients, and at least as frequently among the patients treated by the maximal regimens as among those treated by the other regimens (Subcommittee on clinical trials, unpublished results).

\section{Risk of relapse}

Prior to 1979, a trial of chemotherapy of multibacillary leprosy that involved deliberate cessation of therapy appeared unethical. In that year, however, the results became available of an 8-9 year follow-up in Sungei Buloh of 362 patients with multibacillary leprosy who were 'released from control' - that is, their treatment was stopped - after having been treated with dapsone as monotherapy for 20 years (Waters MFR, Rees RJW et al., unpublished data); the relapse rate averaged only $1 \%$ per year. At the same time, the results were reported (Leiker DL, unpublished data) from Malta of 85 multibacillary patients who, after varying periods of dapsone monotherapy, had been treated with a 2-year course (on average) of daily dapsone and prothionamide, together with daily rifampicin for the first 6 months, and whose treatment had then been stopped. No relapses had been noted in the course of the succeeding 4-5 years. The results of these two studies suggested that relapse of long-or intensively - treated multibacillary leprosy might be far less likely than had been predicted, given the universal presence of persisting $M$. leprae, and the specific immune defect characteristic of these patients.

As soon as it became clear that chemotherapy might safely be stopped after prolonged treatment of multibacillary leprosy with high-quality dapsone monotherapy, or after intensive treatment of much shorter duration, THELEP decided to undertake field trials among large numbers of multibacillary patients of a practical multi-drug regimen, in which efficacy was to be measured in terms of the relapse rate. The THELEP field-trial regimen consists of rifampicin, clofazimine and acedapsone, all administered intermittently, and dapsone administered daily. This regimen is based on the demonstration that single doses of $600 \mathrm{mg}$ rifampicin are equivalent in bactericidal activity to a number of daily doses, ${ }^{21}$ and that rifampicin administered on two consecutive days once monthly is no less effective than rifampicin, $600 \mathrm{mg}$ daily (Rees RJW, et al., unpublished data). Clofazimine has also been shown to be active when administered intermittently. ${ }^{3}$ Acedapsone and the first of two monthly doses of rifampicin and 
clofazimine are administered under full supervision, at the time of a monthly clinic visit, at which time the patient is given the next day's doses of rifampicin and clofazimine and a month's supply of dapsone, all for self-administration. This regimen is to be administered for 2 years to multibacillary patients who are already smear-negative, and until smear-negativity but for a minimum of 2 years to smear-positive patients. After completion of the course of chemotherapy, the patient is to continue under observation, and be given placebo tablets, and randomly-collected urine specimens are to be analysed for the presence of dapsone. Follow-up is to continue for 8 or so years. When relapse is suspected, it is to be verified by biopsy, inoculation of normal mice, and testing the susceptibility of the patient's $M$. leprae to dapsone, clofazimine and rifampicin. Sample-size calculations have shown that, at the end of the trial, information on relapse must be available from at least 200 patients per regimen to permit distinction between an annual relapse rate smaller than 1 per 100 and a measured rate greater than 2 per 100 patients at risk.

The WHO Study Group on the Chemotherapy of Leprosy for Control Programmes has recommended ${ }^{56}$ a similar regimen for the treatment of multibacillary leprosy. In this regimen, rifampicin is administered monthly, clofazimine monthly in one dosage and daily in another, and dapsone daily. The monthly doses are administered under supervision, and the daily doses are unsupervised. This regimen is also undergoing trial in the two THELEP field trials.

Because they include two bactericidal drugs in addition to dapsone, the THELEP field trial regimen and the WHO Study Group regimen should be fully effective in patients whose organisms are already dapsone-resistant, as well as those who have responded well to dapsone monotherapy, but who may well harbour a larger-than-normal subpopulation of dapsone-resistant M. leprae. Thus, no relapses are expected to be caused by the emergence of drug-resistant organisms, and those relapses that do occur should respond readily to retreatment by the same regimen. To prevent the emergence of drug-resistant organisms, combined chemotherapy with bactericidal drugs should be administered for so long as the patient's bacterial population is large enough to contain drug-resistant mutants. If the supplies of rifampicin and clofazimine are limited, they will be used most effectively by intermittent administration, thus prolonging the period during which they may be administered. This is, at least theoretically, much more sound than administering these drugs daily in a much shorter course of combined treatment. Finally, there is an obvious advantage to the supervised administration of drugs in the treatment of leprosy, in which poor compliance of patients with the prescribed treatment regimens so often results in failure of the treatment. ${ }^{14}$ 


\section{References}

1 Ambrose EJ, Antia NH, Khanolkar SR. Uptake of radioactive DOPA by M. leprae. Nature, 1974; 249: 854-5.

${ }^{2}$ Collaborative effort of the U.S. Leprosy Panel (U.S.-Japan Cooperative Medical Science Program) and the Leonard Wood Memorial. Rif ampin therapy of lepromatous leprosy. Amer J Trop Med Hyg, 1975; 24: 475-84.

${ }^{3}$ Collaborative effort of the U.S. Leprosy Panel (U.S.-Japan Cooperative Medical Science Program) and the Leonard Wood Memorial. Spaced clofazimine therapy of lepromatous leprosy. Amer J Trop Med Hyg, 1976; 25: 437-44.

${ }^{4}$ Colston MJ, Hilson GRF, Banerjee DK. The 'proportional bactericide test': a method for assessing bactericidal activity of drugs against Mycobacterium leprae in mice. Lepr Rev, 1978; 49: 7-15.

${ }^{5}$ Committee on Experimental Chemotherapy. Experimental chemotherapy in leprosy. Bull WHO, 1976; 53: 425-33.

${ }^{6}$ Davey TF. Diethyl dithioisophthalate (ETIP or 'Etisul') in the treatment of leprosy: second progress report. Lepr Rev, 1954; 30: 141-52.

7 Doull JA. Clinical evaluation studies in lepromatous leprosy. First series: diasone (diamidin), 4,4'-diaminodiphenyl sulfone, dihydrostreptomycin. Int J Lepr, 1954; 22: 377-402.

${ }^{8}$ Doull JA, Rodriguez JN, Davison AR, Tolentino JG, Fernandez JV. Clinical evaluation studies in lepromatous leprosy. Second series: isoniazid and diasone (diamidin), isoniazid and dihydrostreptomycin, also a pilot trial with streptohydrazid. Int $J$ Le pr, 1957; 25: 173-92.

9 Doull JA, Rodriguez JN, Davison AR, Tolentino JG, Fernandez JV. Clinical evaluation studies in lepromatous leprosy. Third series: nicotinamide and BCG vaccination as supplements to diaminodiphenyl sulf one (DDS). Int $J$ Lepr, 1958; 26: 219-35.

10 Doull JA, Rodriguez JN, Tolentino JG, Fernandez JV. Clinical evaluation studies in lepromatous leprosy: a 24 weeks' study of pyrazinamide-isoniazid therapy. Int J Lepr, 1960; 28: $12-17$.

11 Doull JA, Rodriguez JN, Tolentino JG, Fernandez JV. Clinical evaluation studies in lepromatous leprosy: a 48-weeks' study of cycloserine therapy. Int J Lepr, 1960; 28: 18-21.

12 Doull JA, Rodriguez JN, Tolentino JG, Fernandez JV, Guinto RS, Rivera JN, Mabalay MC. Clinical evaluation studies in lepromatous leprosy. Fourth series: 4-butoxy-4'-dimethylaminodiphenyl thiourea (DPT), amodiaquin, and 4,4'-diaminodiphenyl sulfone (DDS) $2 \cdot 5$ mgm. and $4 \mathrm{mgm}$. per kg. of body weight. Int J Lepr, 1961; 29: 291-317.

13 Doull JA, Tolentino JG, Rodriguez JN, Guinto RS, Rivera JN, Fernandez JV, Mabalay MC. Clinical evaluation studies in lepromatous leprosy. Fifth series: diethyldithioisophthalate (etisul) as a supplement to 4,4'-diaminodiphenyl sulfone (DDS). Int J Lepr, 1965; 33: 186-205.

${ }_{14}$ Ellard GA. Drug compliance in the treatment of leprosy. Lepr Rev, 1981; 52: 201-13.

15 Faget GH, Pogge R C, Johansen FA, Dinan F, Prejean BM, Eccles CG. The promin treatment of leprosy. A progress report. Publ Health Rep, 1943; 58: 1729-41.

16 Gelber RH, Waters MFR, Pearson JMH, Rees RJW, McDougall AC. Dapsone alone compared with dapsone plus rif ampicin in short-term therapy of lepromatous leprosy. Lepr Rev, 1977; 48: 223-9.

17 Guinto RS, Cellona RV, Fajardo TT, de la Cruz EC. Primary dapsone-resistant leprosy in Cebu, Philippines. Int J Lepr, 1981; 49: 427-30.

18 Khanolkar SR. Preliminary studies of the metabolic activity of purified suspensions of Mycobacterium leprae. J Gen Microbiol, 1982; 128: 423-5.

19 Khanolkar Sr, Ambrose EJ, Mahadevan PR. Uptake of 3,4-dihydroxy[ $\left.{ }^{3} \mathrm{H}\right]$ phenylalanine by Mycobacterium leprae isolated from frozen $\left(-80^{\circ} \mathrm{C}\right)$ armadillo tissue. $J$ Gen Microbiol, 1981; 127: $385-9$. 
${ }^{20}$ Levy L, Shepard CC, Fasal P. Clofazimine therapy of lepromatous leprosy caused by dapsone-resistant Mycobacterium leprae. Amer J Trop Med Hyg, 1972; 21: 315-21.

${ }^{21}$ Levy L, Shepard CC, Fasal P. The bactericidal effect of rif ampicin on M. leprae in man: a) single doses of 600, 900 and $1200 \mathrm{mg}$; and b) daily doses of $300 \mathrm{mg}$. Int J Lepr, 1976: 44: 183-7.

22 Lowe J. The chemotherapy of leprosy. Late results of treatment with sulphone, and with thiosemicarbazone. Lancet, 1954; ii: 1065-8.

${ }^{23}$ McRae DH, Shepard CC. Relationship between the staining quality of Mycobacterium leprae and infectivity for mice. Infect Immun, 1971; 3: 116-20.

${ }^{24}$ Meade TW, Pearson JMH, Rees RJW, North WRS. The epidemiology of sulphone-resistant leprosy. Int J Lepr, 1973; 41: 684.

${ }_{25}$ Muir E. Bacteriological changes under DDS treatment of leprosy. Lepr India, 1951; 23: 11626.

${ }^{26}$ Nath I, Prasad HR, Sathish M, Sreevatsa, Desikan KV, Seshadri PS, Iyer CGS. Rapid radiolabeled macrophage culture method for detection of dapsone-resistant Mycobacterium leprae. Antimicrob Ag Chemother, 1982; 21: 26-32.

27 Pattyn SR. A comparison of the bactericidal activity of a series of rifamycins against Mycobacterium leprae. Arzneimit-Forsch, 1982; 32: 15-17.

28 Pattyn SR, Hebrant F. Multivariate analysis of the activity of rifamycins against rapidly growing mycobacteria, to define an in vitro screening model for this activity against Mycobacterium leprae. Arzneimit-Forsch, 1980; 30: 2099-2103.

${ }_{29}$ Pearson JMH. The problem of dapsone-resistant leprosy. Int J Lepr, 1981; 49: 417-20.

30 Pearson JMH, Haile GS, Rees RJW. Primary dapsone resistant leprosy. Lepr Rev, 1977; 48: $129-32$.

${ }^{31}$ Pearson JMH, Pettit JHS. Chemotherapeutic trials in leprosy. 7. Trial of $50 \mathrm{mgm}$. DDS twice weekly in the treatment of lepromatous leprosy. Int J Lepr, 1969; 37: 40-5.

32 Pettit JHS, Rees RJW. Sulphone resistance in leprosy. An experimental and clinical study. Lancet, 1964; ii: 673-4.

${ }^{33}$ Pettit JHS, Rees RJW. Studies on sulfone resistance in leprosy. 2. Treatment with a riminophenazine derivative (B.663). Int J Lepr, 1966; 34; 391-7.

${ }^{34}$ Pettit JHS, Rees RJW. Chemotherapeutic trials in leprosy. 4. Dapsone (DDS) in low dosage in the treatment of lepromatous leprosy. A demonstration pilot study. Int J Lepr, 1967; 35: $140-8$.

${ }^{35}$ Pettit JHS, Rees RJW, Ridley DS. Chemotherapeutic trials in leprosy. 3. Pilot trial of a riminophenazine derivative, B.663, in the treatment of lepromatous leprosy. Int J Le pr, 1967; 35: 25-33.

${ }^{36}$ Portaels F. Unclassified mycobacterial strains susceptible to dapsone isolated from the environment in Central Africa. Int J Lepr, 1980; 48: 330-1.

${ }^{37}$ Prasad HK, Nath I. Incorporation of ${ }^{3} \mathrm{H}$ thymidine in Mycobacterium leprae within differentiated human macrophages. J Med Microbiol, 1981; 14: 279-93.

${ }^{38}$ Rees RJW. Enhanced susceptibility of thymectomized and irradiated mice to infection with Mycobacterium leprae. Nature, 1966; 211: 657-8.

${ }^{39}$ Rees RJW, Valentine RC. The appearance of dead leprosy bacilli by light and electron microscopy. Int J Lepr, 1962; 30: 1-9.

${ }^{40}$ Rees RJW, Valentine RC, Wong PC. Application of quantitative electron microscopy to the study of Mycobacterium lepraemurium and M. leprae. J Gen Microbiol, 1960; 22: 443-57.

${ }^{41}$ Rees RJW, Waters MRF, Pearson JMH, Helmy HS, Laing ABG. Long-term treatment of dapsone-resistant leprosy with rifampicin: clinical and bacteriological studies. Int $J$ Lepr, 1976; 44: 159-69.

42 Ridley DS. A logarithmic index of bacilli in biopsies. 2. Evaluation. Int J Lepr, 1967; 35: 187-93.

${ }^{43}$ Russell DA, Shepard CC, McRae DH, Scott GC, Vincin DR. Acedapsone (DADDS) treatment 
of leprosy patients in the Karimui of Papua New Guinea: status after six years. Amer J Trop Med Hyg, 1975; 24: 485-95.

${ }^{44}$ Sathish M, Nath I. The uptake of ${ }^{3} \mathrm{H}$-thymidine in Mycobacterium leprae inoculated mouse macrophage cultures as a rapid indicator of bacillary viability: factors influencing the specificity of the assay. Int $J$ Lepr, 1981; 49: 187-93.

${ }^{45}$ Seydel JK, Wempe E. Bacterial growth kinetics of ' $M$. lufu' in the presence and absence of various drugs alone and in combination. A model for the development of combined chemotherapy against $M$. leprae. Int J Lepr, 1982; 50: 20-30.

46 Shepard CC. Acid fast bacilli in nasal excretions in leprosy, and results of inoculation of mice. Amer J Hyg, 1960; 71: 147-57.

${ }^{47}$ Shepard CC. The experimental disease that follows the injection of human leprosy bacilli into foot-pads of mice. J Exp Med, 1960; 112: 445-54.

48 Shepard CC. A kinetic method for the study of activity of drugs against Mycobacterium leprae in mice. Int J Lepr, 1967; 35: 429-35.

${ }^{49}$ Shepard CC. Further experience with the kinetic method for the study of drugs against Mycobacterium leprae in mice. Activities of DDS, DFD, ethionamide, capreomycin and PAM 1392. Int J Lepr, 1969; 37: 389-97.

${ }^{50}$ Shepard CC, Chang YT. Effect of several anti-leprosy drugs on multiplication of human leprosy bacilli in foot-pads of mice. Science, 1962; 109: 636-8.

${ }^{51}$ Shepard CC, Chang YT. Activity of antituberculosis drugs against Mycobacterium leprae. Int J Lepr, 1964; 32: 260-71.

52 Shepard CC, Levy L, Fasal P. The death of Mycobacterium leprae during treatment with 4,4'-diaminodiphenylsulfone (DDS). Amer J Trop Med Hyg, 1968; 17: 769-75.

${ }^{53}$ Shepard CC, Levy L, Fasal P. The death rate of Mycobacterium leprae during treatment of lepromatous leprosy with acedapsone (DADDS). Amer J Trop Med Hyg, 1972; 21: 440-5.

${ }^{54}$ Shepard CC, Levy L, Fasal P. Rapid bactericidal effect of rif ampicin on Mycobacterium leprae. Amer J Trop Med Hyg, 1972; 21: 446-9.

55 Shepard CC, Levy L. Fasal P. Further experience with the rapid bactericidal effect of rif ampin on Mycobacterium leprae. Amer J Trop Med Hyg, 1974; 23: 1120-4.

56 Study Group on Chemotherapy of Leprosy for Control Programmes. WHO Tech. Rep. Series 675. Geneva, 1982.

57 Subcommittee on Clinical Trials of the Chemotherapy of Leprosy (THELEP) Scientific Working Group of the UNDP/World Bank/WHO Special Programme for Research and Training in Tropical Diseases. THELEP controlled clinical trials in lepromatous leprosy. Lepr Rev, 1983; 54:

58 Subcommittee on Clinical Trials of the Chemotherapy of Leprosy (THELEP) Scientific Working Group of the UNDP/World Bank/WHO Special Programme for Research and Training in Tropical Diseases. Primary resistance to dapsone in Bamako and Chingleput. Lepr Rev, 1983; 54:

59 Tolentino JG. Results of six months supplementary treatment of lepromatous patients with Mycobacterium marianum vaccine. Int J Lepr, 1957; 25: 351-5.

${ }^{60}$ Waters MFR. Chemotherapeutic trials in leprosy. 1. Comparative trial of macrocyclon plus dapsone and dapsone alone in the treatment of lepromatous leprosy. Lepr Rev, 1963; 34: 173-92.

${ }^{61}$ Waters MFR. Chemotherapeutic trials in leprosy. 6. Pilot study of the riminophenazine derivative B.663 in low dosage (100 mgm. twice weekly) in the treatment of lepromatous leprosy. Int J Lepr, 1968; 36: 391-9.

62 Waters MFR, Pettit JHS. Chemotherapeutic trials in leprosy. 2. Comparative trial of dapsone plus ditophal (etisul) and dapsone alone in the treatment of lepromatous leprosy. Int J Lepr, 1965; 33: 280-96. 
${ }^{63}$ Waters MFR, Rees RJW. Changes in the morphology of Mycobacterium leprae in patients under treatment. Int J Le pr, 1962; 30: 266-77.

${ }^{64}$ Waters MFR, Rees RJW, McDougall AC, Weddell AGM. Ten years of dapsone in lepromatous leprosy: clinical, bacteriological and histological assessment and the finding of viable leprosy bacilli. Lepr Rev, 1974: 45: 288-98.

65 World Health Organization. A Guide to Leprosy Control. Geneva, 1980. 